\title{
Can platelet-rich plasma be used safely in intra-abdominal operations?
}

\author{
Kaya $\mathrm{F}^{1}$, Kismet $\mathrm{K}^{1}$, Ozer $\mathrm{H}^{1}$, Soylu $\mathrm{VG}^{1}$, Duymus $\mathrm{ME}^{1}$, Akgun $\mathrm{YA}^{1}$, Demir $\mathrm{A}^{1}$, Celepli $\mathrm{P}^{2}$, \\ Guclu T ${ }^{3}$, Bolat $\mathrm{S}^{3}$, Ertas $\mathrm{E}^{1}$
}

Ankara Education and Research Hospital, Department of General Surgery, Ankara, Turkey. kemalkismet@yahoo.com

\section{ABSTRACT}

OBJECTIVES: In this study, it was aimed to investigate whether or not platelet-rich plasma (PRP) causes intraabdominal adhesions and therefore, whether or not PRP can be used safely in intra-abdominal operations. METHODS: Of the total of 35 animals, 5 were used as donors for the preparation of platelet-rich plasma (PRP). The surgical procedures were performed on the remaining 30 animals. These rats were randomized and divided into 3 groups of 10. In Group 1, no adhesion induction was performed. Adhesion was induced by cecal abrasion and peritoneal resection model in Groups II and IIII. In Group 2, no treatment was given. In Group 3, 1 cC PRP was applied on the cecum. The rats were sacrificed on postoperative day 21.

RESULTS: According to adhesion scores, the difference between the sham and PRP groups was not statistically significant. There was also no significant difference between the control and PRP groups, but the adhesion scores in the PRP group was lower than those in the control group. On histopathological evaluation, the difference between the sham and PRP groups was not statistically significant. There was also no significant difference between the control and PRP groups, but the average fibrosis and inflammation scores in the PRP group were lower than those in the control group.

CONCLUSION: The results of the present study have demonstrated that PRP neither reduced nor exacerbated postoperative adhesions. Thus, PRP can be used safely in experimental and clinical studies where it will be applied intra-abdominally (Tab. 2, Fig. 3, Ref. 11). Text in PDF www.elis.sk.

KEY WORDS: platelet rich plasma, intra-abdominal operations, adhesions, rats.

\section{Introduction}

Platelets are nonnuclear cellular fragments derived from megakaryocytes in the bone marrow; they are specialized secretory elements that release the contents of their intracellular granules in response to activation. Platelets contain a great variety of protein molecules, among which are a high rate of signaling, membrane proteins, protein processing, cytoskeleton regulatory proteins, cytokines, and other bioactive peptides that initiate and regulate basic aspects of wound healing. It is known that more than 300 proteins are released by human platelets in response to thrombin activation. In addition to their well-known function in hemostasis, platelets also release substances that promote tissue repair, angiogenesis, and inflammation. Furthermore, platelets induce the migration and adherence of bone-marrow-derived cells to sites of angiogenesis and induce differentiation of endothelial-cell progeni-

\footnotetext{
${ }^{1}$ Ankara Education and Research Hospital, Department of General Surgery, Ankara, Turkey, ${ }^{2}$ Aksaray State Hospital, Department of Pathology, Aksaray, Turkey, and ${ }^{3}$ Ankara Education and Research Hospital, Department of Biochemistry, Ankara, Turkey

Address for correspondence: K. Kismet, S.B. Ankara Egitim ve Arastirma Hastanesi Genel Cerrahi Klinigi, Ulucanlar, Ankara, Turkey. Phone: +90.312 .5953885 , Fax: +90.312 .3633396$
}

tors into mature endothelial cells. Platelet-derived Growth Factors (GFs) are biologically active peptides that enhance tissue repair mechanisms such as angiogenesis, extracellular matrix remodeling, and cellular effects as stem cell recruitment, chemotaxis, cell proliferation, and differentiation. Platelet-rich plasma (PRP) is used in a variety of clinical applications, based on the premise that higher GF content should promote better healing. Platelet derivatives represent a promising therapeutic modality, offering opportunities for the treatment of wounds, ulcers, soft-tissue injuries, and various other applications in cell therapy (1). Apart from these indications, it has also been used to obtain improved strength in colonic and intestinal anastomoses, and to augment wound healing in open abdomen (2-6).

Adhesion formation following surgery remains an almost inevitable consequence of most abdominal procedures. Studies have found the incidence of adhesions to be as high as $95 \%$. Advances in surgical technique, such as the use of laparoscopic surgery, can help minimize the probability of adhesion formation, although the significant morbidity and cost associated with adhesion-related disorders continues to be highly prevalent. Whilst many methods have been employed in an attempt to reduce the formation of adhesions, no single approach has been wholly satisfactory. Intraabdominal adhesions cause adhesive small bowel obstruction, volvulus or chronic abdominal pain. In female patients, adhesions 
Tab. 1. Adhesion Scoring System.

\begin{tabular}{|c|c|c|c|}
\hline SCORE & EXTENT & APPEARANCE & STRENGTH \\
\hline 0 & No & No & No \\
\hline 1 & Less than $25 \%$ & Filmy, avascular & Separated easily \\
\hline 2 & Between $25-50 \%$ & Dense, avascular & Separated by traction \\
\hline 3 & Between $50-75 \%$ & Dense,capillary vascularization & Sharp dissection needed for seperation \\
\hline 4 & More than $75 \%$ & Dense, vascular & Sharp dissection needed for seperation \\
\hline
\end{tabular}

within the pelvis may result in infertility, ectopic gestation and chronic pelvic pain. In patients with secondary problems from adhesions, future surgery (for abdominal pathology other than adhesions) may be complicated or prolonged. In such cases, the number and density of adhesions will have a direct impact on the second surgical procedure (7).

As previously mentioned, there have been various studies of PRP used in intra-abdominal procedures. Since postoperative adhesions constitute the major problem of intra-abdominal procedures, a product which will be used in intra-abdominal procedures should not exacerbate adhesions. In this study, it was aimed to investigate whether or not PRP causes intra-abdominal adhesions and therefore, whether or not PRP can be used safely in intra-abdominal operations.

\section{Materials and methods}

\section{Animals}

Thirty-five Wistar-Albino female rats, weighing $225 \pm 25 \mathrm{~g}$, were allowed to adapt to laboratory conditions for 1 week before experimental use. The animals had free access to water and standard laboratory chow. They were kept individually in wire cages at a constant temperature $\left(21 \pm 2{ }^{\circ} \mathrm{C}\right)$ under a 12-hour light-dark cycle. Twelve hours before anesthesia, the animals were deprived of food but had free access to water until 2 hours before anesthesia. No enteral or parenteral antibiotics were administered at any time. Rats that died during the experiment were excluded from the study and no new rats were included. The procedures in this experimental study were performed in accordance with the National Guidelines for The Use and Care of Laboratory Animals and approved by Animal Ethics Committee of Ankara Research and Training Hospital (The Name of the Chairperson of the Ethic Committee: Prof. Dr. Nevres Aydogan, The Protocol Number: 239, The Date of Approval: 27.12.2013).

\section{Study groups}

Of the total of 35 animals, 5 were used as donors for the preparation of platelet-rich plasma (PRP). The surgical procedures were performed on the remaining 30 animals. These rats were randomized and divided into 3 groups of 10. All animals were anesthetized with an intramuscular injection of $50 \mathrm{mg} / \mathrm{kg}$ ketamine hydrochloride (Ketalar ${ }^{\circledR}$, Parke-Davis, Istanbul) and 5 $\mathrm{mg} / \mathrm{kg}$ xylazine (Rompun ${ }^{\circledR}$, Bayer, Istanbul).

The abdomen was shaved and prepared with povidone-iodine. Under sterile conditions, a midline laparotomy was performed. The cecum was abraded with sterile gauze until a subserosal hemor- rhage developed. A 1x1 cm patch of peritoneum located opposite the cecal abrasion was completely dissected. In Group 1 (sham group), laparotomy was performed, the cecum was removed from the abdomen, and replaced in the abdomen without adhesion induction. In Group 2 (control group), adhesion induction was performed and no treatment was given. In Group 3 (PRP group), after adhesion induction, $1 \mathrm{cc}$ PRP was applied on the cecum. Animals were allowed access to food and water after the operation. All operations were performed by the same surgeon. The rats were sacrificed on postoperative day 21 . Adhesions were classified by a surgeon blinded to the groups, according to a scoring system based on evaluation of the appearance, extent and strength of the adhesions (Tab. 1). Peritoneal and intestinal tissue samples were taken for histopathological evaluation.

\section{Preparation of $P R P$}

The 5 rats selected as donors for the preparation of PRP underwent no surgical procedure. These rats were sacrificed by high dose of ketamine hydrochloride in injection and whole body blood was taken from the inferior vena cava. The $10 \mathrm{~mL}$ of blood obtained from the donor rats was put into biochemistry tubes containing citrate-phosphate-dextrose (CPD) solution, then centrifuged for 10 min at $1500 \mathrm{rpm}$. After centrifugation, three layers were obtained: the top layer including plasma with a light yellow color, the bottom layer mainly including erythrocytes, and a thin intermediate layer between these layers including platelets and white blood cells (buffy coat). The plasma layer at the top was withdrawn into another centrifugation tube and subjected to second centrifugation for $10 \mathrm{~min}$ at $3000 \mathrm{rpm}$, after which two layers were obtained; the upper layer was platelet-poor plasma (PPP) and the lower was PRP. The PPP and PRP were carefully separated into different tubes, and PRP was used in this study.

\section{Histopathological evaluation}

The histopathological examinations consisting of light microscope analyses were carried out at the Pathology Department of Aksaray State Hospital. The samples obtained from the abraded cecal tissue and the adjacent peritoneal tissue were being fixed in $10 \%$ neutral buffered formalin solution for 2 days. Tissues were washed in running water, and dehydrated with increasing concentrations of ethanol (50\%, $75 \%, 96 \%$ and $100 \%)$. After dehydration, specimens were placed in xylene to obtain transparency and embedded in paraffin. The embedded tissues were cut into $5 \mu \mathrm{m}$-thick sections and stained with hematoxylin and eosin and trichrome. Histopathological examinations were performed by a pathologist blinded to the study groups. The samples stained 
Tab. 2. Mean adhesion scores and pathological scores of the groups.

\begin{tabular}{lccc}
\hline GROUPS & MEAN ADHESION SCORES & FIBROSIS & INFLAMMATION \\
\hline Group 1 (Sham) & $1.80 \pm 1.02 *$ & $0.30 \pm 0.15^{*}$ & $0.40 \pm 0.21^{*}$ \\
Group 2 (Control) & $5.70 \pm 2.73$ & $1.60 \pm 0.79$ & $1.80 \pm 0.85$ \\
Group 3 (PRP) & $3.00 \pm 1.51$ & $0.66 \pm 0.32$ & $0.88 \pm 0.42$ \\
\hline
\end{tabular}

* Significantly different from control group

with hematoxylin and eosin were examined for inflammation, and the presence of fibrosis was evaluated in the hematoxylin/ eosinand trichrome-stained samples using a semi-quantitative scoring system. The scoring systems were as follows:

Inflammation Scoring System: Score 0: No inflammation, Score 1: Giant cells, lymphocytes, and plasma cells; Score 2: Giant cells, plasma cells, eosinophiles, and neutrophils; Score 3: Inflammatory cell infiltration and microabscess formation.

Fibrosis Scoring System: Score 0: No fibrosis; Score 1: Mild; Score 2: Moderate, Score 3: Severe.

\section{Statistical analysis}

Data analysis was performed using the Statistical Package for Social Sciences (SPSS) version 15.0 for Windows (SPSS Inc, Chicago, IL). All variables were found to be normally distributed about the mean. Data were presented as mean \pm SD. Differences between the groups were evaluated by one-way analysis of variance (ANOVA) or Kruskal-Wallis variance analysis, as appropriate. When the $\mathrm{p}$ values from the variance analysis were statistically significant, the Tukey honestly significant difference (HSD) or Mann-Whitney U multiple comparison test was used to determine which group was different from the others. A value of $p<0.05$ was considered statistically significant.

\section{Results}

General

One rat from the PRP group (Group 3) died during the study. This rat was excluded from the study and no new rat was included. The remaining rats were sacrificed on postoperative day 21 .

\section{Adhesion Scores}

The mean adhesion scores are summarized in Table 2. There was a significant difference between the sham group and the control group $(p=0.013)$. The difference between the sham and PRP groups was not statistically significant $(p>0.05)$, but the average adhesion score in the sham group was lower than that in the PRP group. There was also no significant difference between the control and PRP groups ( $p>0.05)$, but the average adhesion score in the PRP group was lower than that in the control group (Tab. 2).

\section{Histopathological results}

The histological findings of the groups are represented in Figures 1-3. The mean pathological scores of the groups are summarized in Table 2. The difference between the sham and control groups was statistically significant $(\mathrm{p}=0.009$ for fibrosis, and $\mathrm{p}$ $=0.005$ for inflammation). Although the difference between the sham and PRP groups was not statistically significant $(p>0.05)$, the
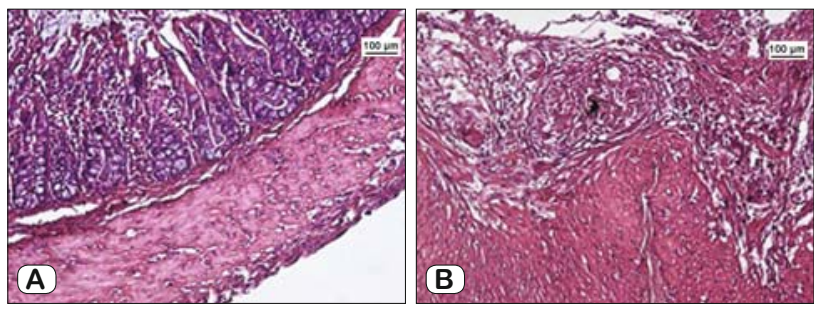

Fig. 1. (Inflammation score: 1) (H\&E). A) Mild serosal inflammation. B) Foreign body-type multinucleated giant cells and a small number of lymphocytes and plasma cells.

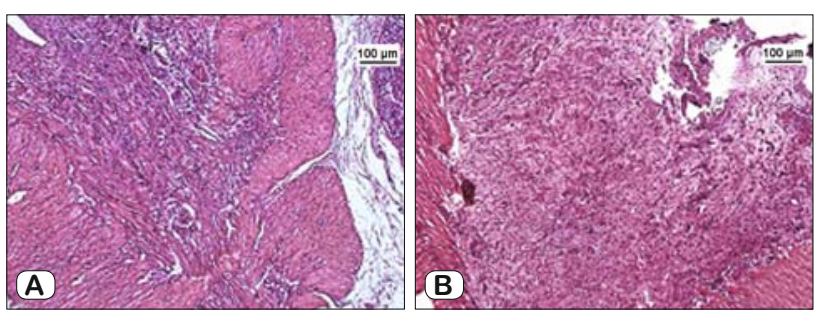

Fig. 2. Moderate inflammation (Inflammation score: 2) (H\&E). A) An inflammatory focus is seen near the serosal surface of the colon wall. B) The foreign body-type multinucleated giant cells, eosinophils, lymphocytes and plasma cells.
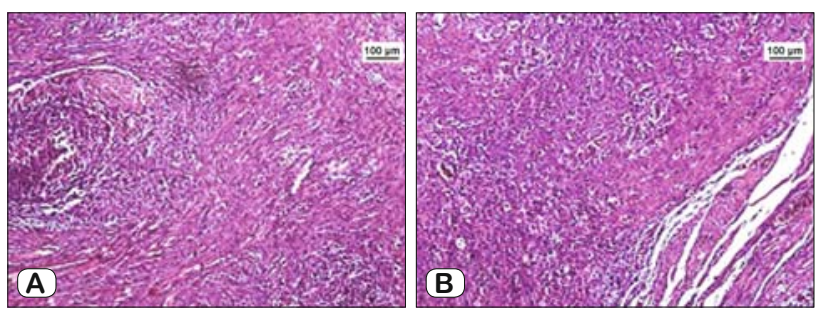

Fig. 3. Severe inflammation (Inflammation score: 3) (H\&E). A) An inflammatory focus is seen near the serosal surface of the colon wall. B) The foreign body-type multinucleated giant cells, eosinophils, lymphocytes and plasma cells.

average fibrosis and inflammation scores in the sham group were lower than that in the PRP group. There was also no significant difference between the control and PRP groups $(p>0.05)$, but the average fibrosis and inflammation scores in the PRP group were lower than that in the control group. The pathological scores and findings were in accordance with the adhesion scores.

\section{Discussion}

Abdominal surgery can cause adhesions between tissues and organs. It has been reported that up to $93 \%$ of patients who have undergone one or more previous surgeries have intra-abdominal 
adhesions (1). Postsurgical adhesions are a consequence of injured tissue surfaces (following incision, cauterization, suturing or other trauma) fusing together to form scar tissue. Postsurgical adhesions severely affect the quality of life of millions of people worldwide, causing small-bowel obstruction, difficult revision surgery, chronic abdominal and pelvic pain, and female infertility. Re-operating through the previous wound can be extremely difficult, risky, and potentially dangerous. Also, adhesiolysis extends operating time, anesthesia, and recovery time placing the patient at additional risk of blood loss, visceral damage including injury to the bladder, enterocutaneous fistulas, and resection of damaged bowel (8).

The first data on induction of adhesions in an animal model was published in 1889, and the intervening 120 years have seen extensive studies in vitro and in vivo. Nevertheless, the literature contains neither an official definition of adhesions nor a recognized standardized classification for objective assessment of their extent and severity. Accordingly, study findings have often been imprecise and do not lend themselves to adequate interpretation. By the same token, there is a lack of clinically oriented guidelines for diagnosis, treatment and options for reduction of adhesions (9).

Intra-abdominal adhesions may be congenital or acquired. Congenital adhesions arise during physiological organogenesis or can be traced back to abnormal embryonal development of the abdominal cavity. They are usually asymptomatic and are diagnosed incidentally. Postmortem examinations of patients who have not undergone surgery have identified post-inflammatory adhesions in $28 \%$ of cases. These are caused by intra-abdominal inflammation or can be attributed to endometriosis, peritonitis, radiotherapy, or long-term peritoneal dialysis (9).

Intra-abdominal adhesions arising from a surgical procedure can cause complications decades later. Patient symptoms include meteorism, irregular bowel movements, chronic abdominal pain, digestive disorders, infertility, and intestinal obstruction, and often fail to be associated with their cause. In contrast to congenital or post-inflammatory adhesions, which are mostly asymptomatic, postoperative adhesions cause $40 \%$ of all cases of intestinal obstruction. Particularly colectomy, which involves a large peritoneal incision, carries an $11 \%$ cumulative risk of intestinal obstruction within the first year postoperatively. Adhesions are also responsible for $15 \%$ to $20 \%$ of all cases of secondary female infertility. On the other hand, chronic lower abdominal pain severely impairs the quality of life of those affected and forms the indication for $30 \%$ to $50 \%$ of all laparoscopies and $5 \%$ of hysterectomies (9). Numerous attempts have been made to prevent or reduce the incidence of peritoneal adhesions, but with limited success (8).

Postoperative adhesions develop as a result of wound healing and are influenced by various factors such as the complexity of operation, the extent of peritoneal trauma, accompanying illness (e.g., diabetes), poor nutritional status, intra-abdominal placement of foreign bodies (e.g. meshes), excessive coagulation with tissue necrosis, accompanying bacterial infection, dehydration owing to high insufflation pressure and compression of capillary flow during laparoscopy, exposure to foreign material (e.g., glove powder, suture materials, etc.), mesothelial dehydration and abrasion from use of dry abdominal drapes, and some drugs used intra-abdominally (9).
Strategies to reduce adhesion formation include improving surgical techniques, optimizing laparoscopy conditions, using pharmacology interventions targeted at the inflammatory response and/or fibrin deposition, and using agents that provide a physical barrier to adhesion formation. While these strategies have provided some success, none have yet proved totally successful in abolishing adhesions (8).

Since intra-abdominal adhesions cause serious problems, it is essential that the drugs to be used intra-abdominally should not cause adhesions.

Platelet concentrates for topical and infiltrative use are used or tested as surgical adjuvants or regenerative medicine preparations in most medical fields, particularly in sports medicine and orthopedic surgery. These concentrates are primarily blood extracts obtained after various stages of processing of a whole blood sample, mostly through centrifugation. The objective of the processing is to separate the blood components in order to discard the unusable elements (mostly the red blood cells, heavy and easily separated) and to gather and concentrate the elements that may be of use in therapeutic applications (fibrinogen/fibrin, platelets, growth factors, leukocytes and other forms of circulating cells, in solution in liquid plasma). In short, all these products, whatever the technique used, are extracts of the blood circulating tissue. They are tissues themselves, and not pharmaceutical preparations. These preparations are used on a surgical or wounded site in order to stimulate, improve and accelerate healing (10).

Platelet-derived factors have been extensively used for clinical and surgical applications requiring tissue regeneration. The rationale for the widespread use of platelet derivatives in the healing process is due to the abundance and accessibility of critical growth factors and other signaling molecules in platelets. Overall, platelet derivatives could be a promising therapeutic tool in many areas such as periodontal, oral, maxillofacial, orthopedic bone and tendon defects, and dermatological procedures such as chronic wounds. Despite numerous studies conducted in recent years in several medical fields, important questions remain unresolved, particularly with regard to the timing of the therapy and to the actual impact of the use of platelet derivatives on wound rehabilitation, pain reduction, functional recovery, antibacterial activity, and cancer development (11).

Apart from the above-mentioned types of studies, PRP has also been used in intra-abdominal operations. Yol et al (2) compared the effects of PRP and tissue adhesive Bioglue ${ }^{\circledR}$ on tissue maturation and wound healing in experimental colonic anastomosis. Bursting pressures and hydroxyproline levels were found to be statistically higher in the PRP group than in the control and Bioglue groups. Histopathologically, there was less inflammatory cell infiltration, intensive fibroblast development, and rich collagen production in the PRP group. It was concluded that PRP may be used in colon anastomosis, especially in patients with impaired wound healing, to obtain better anastomotic strength (2). In another two studies, the effects of PRP on intestinal anastomoses were evaluated. Fresno et al. (4) investigated the effects of PRP on intestinal healing following intestinal anastomosis using histology and measurement of the anastomotic breaking strength as end points in pigs. The activated 
form of PRP was used and PRP application appeared to increase granulation tissue and fibrosis, but was not found to influence anastomotic breaking strength. In another intestinal anastomotic model, Yamaguchi et al. (3) also investigated the role of PRP on intestinal anastomotic healing. Various other studies have also evaluated the effects of PRP on colonic anastomotic healing and wound healing after open abdomen $(5,6)$.

In brief, PRP has been used in various experimental studies intra-abdominally. Since the positive effects of PRP on anastomosis has been shown in most of these studies and PRP has become a popular natural concentrate for wound healing and anastomotic healing, the authors of the present study predict that PRP will be used in either experimental or clinical studies widely in the future. Therefore, this study was planned to evaluate whether or not PRP exacerbates intra-abdominal adhesions as it is extremely important that material used intra-abdominally should not cause adhesions.

The results of the present study showed no statistically significant difference between the mean adhesion scores and histopathological findings of the PRP group and the other groups. Although the difference between the sham and PRP groups was not statistically significant, the average adhesion score of the sham group was lower than that in the PRP group. There was also no significant difference between the control and the PRP groups ( $p>0.05$ ), but the average adhesion score in the PRP group was lower than that in the control group. The pathological scores and findings were in accordance with the adhesion scores.

In conclusion, the results of the present study have demonstrated that PRP neither reduced nor exacerbated postoperative adhesions. Thus, PRP can be used safely in experimental and clinical studies where it will be applied intra-abdominally.

\section{Learning points}

- PRP neither reduced nor exacerbated postoperative adhesions.

- PRP can be used safely in experimental and clinical studies where it will be applied intra-abdominally.

\section{References}

1. Sánchez-González DJ, Méndez-Bolaina E, Trejo-Bahena NI. Platelet-rich plasma peptides: key for regeneration. Int J Pept 2012: 532519.

2. Yol S, Tekin A, Yilmaz H et al. Effects of platelet rich plasma on colonic anastomosis. J Surg Res 2008; 146: 190-194.

3. Yamaguchi R, Terashima H, Yoneyama S, Tadano S, Ohkohchi N. Effects of platelet-rich plasma on intestinal anastomotic healing in rats: PRP concentration is a key factor. J Surg Res 2012; 173: 258-266.

4. Fresno L, Fondevila D, Bambo O, Chacaltana A, García F, Andaluz A. Effects of platelet-rich plasma on intestinal wound healing in pigs. Vet J 2010; 185: 322-327.

5. Zhou B, Ren J, Ding C et al. Protection of colonic anastomosis with platelet-rich plasma gel in the open abdomen. Injury 2014; 45: 864-868.

6. Zhou B, Ren J, Ding C et al. Rapidly in situ forming platelet-rich plasma gel enhances angiogenic responses and augments early wound healing after open abdomen. Gastroenterol Res Pract 2013: 926764.

7. Lauder CI, Garcea G, Strickland A, Maddern GJ. Abdominal adhesion prevention: still a sticky subject? Dig Surg 2010; 27: 347-358.

8. Ergul E, Korukluoglu B. Peritoneal adhesions: facing the enemy. Int J Surg 2008; 6: 253-260.

9. Brüggmann D, Tchartchian G, Wallwiener M, Münstedt K, Tinneberg HR, Hackethal A. Intra-abdominal adhesions: definition, origin, significance in surgical practice, and treatment options. Dtsch Arztebl Int 2010; 107: 769-775.

10. Dohan Ehrenfest DM, Andia I, Zumstein MA, Zhang CQ, Pinto NR, Bielecki T. Classification of platelet concentrates (Platelet-Rich Plasma-PRP, Platelet-Rich Fibrin-PRF) for topical and infiltrative use in orthopedic and sports medicine: current consensus, clinical implications and perspectives. Muscles Ligaments Tendons J 2014; 4: 3-9.

11. De Pascale MR, Sommese L, Casamassimi A, Napoli C. Platelet Derivatives in Regenerative Medicine: An Update. Transfus Med Rev 2015; 29: 52-61.

Received March 14, 2016. Accepted April 1, 2016. 